\title{
Temperature dependence of strain-induced crystallization in natural rubber: On the presence of different crystallite populations
}

\author{
Nicolas Candau a, b, Rabia Laghmach b, c, Laurent Chazeau a, b, *, Jean-Marc Chenal a, b, \\ Catherine Gauthier ${ }^{\mathrm{d}}$, Thierry Biben ${ }^{\mathrm{c}}$, Etienne Munch ${ }^{\mathrm{d}}$ \\ a Université de Lyon, CNRS, France \\ ${ }^{\mathrm{b}}$ MATEIS, INSA-Lyon, CNRS UMR5510, F-69621, France \\ c Institut Lumière Matière, UMR5306 CNRS, Université Claude Bernard Lyon 1, 69622 Villeurbanne Cedex, France \\ ${ }^{\mathrm{d}}$ Manufacture Française des Pneumatiques Michelin, Centre de technologies, 63040 Clermont Ferrand Cedex 9, France
}

The effect of the temperature on strain induced crystallization (SIC) of natural rubber (NR) is studied by in situ wide angle X-rays scattering (WAXS) experiments performed along different thermo-mechanical paths (cyclic deformation at different temperatures or crystallization and melting in the deformed state). The crystallinity index (CI), the average crystallite size $\left(L_{200}\right)$ and the average volume of the crystallites $(V)$ increase (decrease) similarly if the sample is cooled (heated) in the deformed state or stretched (unstretched) at fixed temperature. These experiments are analyzed through a thermodynamic description assuming that SIC is controlled by successive nucleation of crystallite populations. Such description predicts that for a stretching ratio $\lambda$ above (below) 4 and a temperature above (below) room temperature, crystallization first occurs in chains with the highest (lowest) network chain density, leading to the smallest (largest) crystallites.

\section{Introduction}

Temperature is one of the main environmental parameters that control strain-induced crystallization (SIC) in Natural Rubber (NR). This is particularly important in tire application, where the material is usually subjected to severe loadings and thermal conditions (e.g. air temperature variations and self-heating). Some authors reported that the rubber ability to crystallize under strain is strongly enhanced - in terms of kinetics and value of crystallinity - when the temperature is cooled down below room temperature [1-4]. In such studies, temperature is usually fixed at $-25^{\circ} \mathrm{C}$, which corresponds to the optimal temperature of crystallization of NR in undeformed state [5-7]. The final crystalline state is hence the resulting combination of thermal-induced crystallization (TIC) and SIC, leading to the formation of shish-kebab structure. The "shish" ( $\gamma$ lamellae) is formed during the stretching and the "kebab" ( $\alpha$ or $\beta$ lamellae) during the cooling [5-8]. Conversely, when the

\footnotetext{
* Corresponding author. Bâtiment Blaise Pascal, $5^{\text {ème }}$ étage, 7 avenue Jean Capelle, 69621 Villeurbanne, France. Tel.: +33 (0)4 72438357.

E-mail address: laurent.chazeau@insa-lyon.fr (L. Chazeau).
}

temperature of a sample maintained in a stretched state is raised above room temperature, its crystalline phase melts [9-12]. According to various authors, an increase of the temperature leads to an increase of the stretching ratio at SIC onset $\left(\lambda_{c}\right)$, an increase of the melting stretching ratio $\left(\lambda_{\mathrm{m}}\right)$ but also a slight decrease of their difference $[9,12]$. Such difference may be seen as a "superstraining" effect which refers to the classical and well known supercooling effect met in thermal crystallization. Nevertheless, to the authors' knowledge, no investigation was reported on the effect of the temperature on the morphology of the crystallites induced by strain, neither on the effect of a temperature increase on the morphology of crystallites of a constantly stretched rubber. For this reason, no study proposed a complete thermodynamic description of SIC of NR when the sample is submitted to different thermomechanical paths (cyclic deformation at different temperatures or crystallization and melting in the deformed state). In particular, the respective contributions of entropic energy (so-called strain energy), enthalpic energy (temperature dependent) and surface energy (related to crystallite size) on SIC and melting has never been deeply discussed.

This study is thus dedicated to the understanding of the temperature effects on the strain-induced crystallization and melting of 
natural rubber. First, general thermodynamic equations, physical and crystalline parameters necessary to quantify the SIC and melting process, are discussed. The crystallinity index and the crystallite morphology (nucleus and saturated crystallite size) evaluated from in situ WAXS experiments performed along various thermo-mechanical paths (temperature and stretching ratio) are then measured. The experiments are finally compared to the model predictions.

\section{Experimental}

\subsection{Materials}

A crosslinked unfilled NR, obtained by vulcanization of NR gum is considered in this work with the following recipe: $100 \mathrm{phr}$ of rubber gum which is a Technically Specified Rubber (TSR20) provided by Michelin Tire Company, 2 phr of stearic acid (SA), 1.5 phr of zinc oxide (ZnO), 3 phr of 1,3-dimethylbutyl-N'-phenyl-P-phenylenediamine (6PPD), $1.9 \mathrm{phr}$ of cyclohexylbenzothiazole-2sulfenamide (CBS) and $1.2 \mathrm{phr}$ of sulfur (where phr means g per $100 \mathrm{~g}$ of rubber). The material has been processed following the Rauline patent [13]. First, the gum is introduced in an internal mixer and sheared for $2 \mathrm{~min}$ at $60^{\circ} \mathrm{C}$. Then, the vulcanization recipe is added and the mix is sheared for 5 min. Afterward, the material is sheared in an open mill for five minutes at $60^{\circ} \mathrm{C}$. Sample sheets are then obtained by hot pressing at $170{ }^{\circ} \mathrm{C}$ during $13 \mathrm{~min}$. Dumbbellshaped samples, with a $6 \mathrm{~mm}$ gauge length $\left(l_{0}\right)$ and $0.8 \mathrm{~mm}$ thickness, are machined. The number density of the elastically effective subchains (so-called hereafter average network chain density $\nu$ ) was estimated from the swelling ratio in toluene and from the Flory-Rehner equation [14] and found equal to $1.4 \times 10^{-4} \mathrm{~mol} \mathrm{~cm}^{-3}$. This density is tuned so that (i) it promotes the development of strain induced crystallization [15] and (ii) it is high enough to avoid an inverse yield effect [11]. In order to avoid microstructure modification during the different mechanical tests, i.e. an uncontrolled Mullins effect, the samples are stretched four times up to stretching ratio $(\lambda=7)$ higher than the maximum stretching ratio reached during the in situ cyclic tests $(\lambda=6)$. The stretching ratio (so-called nominal or engineering stretching ratio) is defined as the sample length 1 divided by the initial sample length $l_{0}$.

\subsection{In situ WAXS analysis}

The in situ WAXS experiments are carried out on the D2AM beamline of the European Synchrotron Radiation Facility (ESRF). The X-ray wavelength is $1.54 \AA$. Tests are performed in a temperature-controlled chamber, which enables to submit the samples to more or less complex thermo-mechanical history. The following tests are performed: (i) stretching at a constant strain rate $\left(4.2 \times 10^{-3} \mathrm{~s}^{-1}\right)$ and heating in the deformed state, (ii) stretching after pre-heating (from room temperature to $80^{\circ} \mathrm{C}$ ), (iii) thermal cooling in the deformed state after stretching at high temperature (above room temperature). Cooling rate is measured but not controlled. It can be roughly estimated equal to $2{ }^{\circ} \mathrm{C} \mathrm{min}-1$.

The two-dimensional (2D) WAXS patterns are recorded by a CCD camera (Princeton Instrument). The beam size is small enough $(300 \mu \mathrm{m} \times 300 \mu \mathrm{m})$ to avoid superimposition with the scattered signal. The background, (i.e. air scattering and direct beam intensities) is properly measured in absence of any sample. It can then be subtracted to the total intensity scattered in the presence of the rubber sample. The corrected scattering intensity is finally normalized by the thickness and the absorption of the sample. Each scattering pattern is integrated azimuthally. The deconvolution of the curve $I=f(2 \theta)$ enables the extraction of the intensity at the peak top and the width at half height of each crystalline peak and the intensity at the peak top of the amorphous phase. The crystallinity index $\mathrm{CI}$ is then determined as follows [16]:

$C I=\frac{I_{a 0}-I_{a \lambda}}{I_{a 0}}$

where $I_{a 0}$ and $I_{a \lambda}$ are the intensity of the amorphous phase at the peak top in the unstretched state and the stretched state, respectively. The average crystallite sizes $L_{\mathrm{hkl}}\left(L_{200}, L_{102}\right.$ and $\left.L_{002}\right)$ in the direction normal to the $(h k l)$ planes, are estimated from the Scherrer equation:

$L_{h k l}=\frac{K \lambda_{w}}{\beta_{1 / 2} \cos \theta}$

where $\lambda_{\mathrm{w}}$ is the wavelength and $\theta$ is the Bragg angle. In this study, each crystalline peak is fitted with a Lorentzian function in which the width at half-height is $\beta_{1 / 2}$. According to the parameters chosen for the fit of the experimental peak, the K value is 0.64 [17]. In order to measure the average crystallite size in the stretching direction $L_{002}\left(c_{1}\right)$, the tensile test machine is tilted by an angle around $10^{\circ}$. $L_{002}$ only weakly depends on the stretching ratio (refer to Ref. [18]) and is taken equal to $85 \AA$. We also checked that this value remains the same whatever the temperature of the test. In the same study [18], an empiric relationship between the crystallites lateral sizes has been established: $b_{1} \sim a_{1} / 3$ with $L_{200}=a_{1}$ and $L_{020}=b_{1}$. The $b_{1}$ value is deduced from the measurement of $L_{120}$. Given that the angle between the plans (120) and (020) is equal to $19^{\circ}, b_{1}\left(L_{020}\right)$ is equal to $0.94 L_{120}$. The average volume of the crystallites is then defined by $\mathrm{V}=0.94 L_{120} L_{200} L_{002}$ and is therefore proportional to $L_{200}^{2}$.

\section{General equations and parameters}

\subsection{Energetic contributions for SIC}

The classical theory of phase transition [19] is used to describe strain induced crystallization and melting. Crystallization process is assumed to occur in conditions close to the so-called quasi-static ones (rate of crystallization higher than the strain rate or cooling rate). Consequently, the crystal is assumed to grow spontaneously - compared to the time scale of the experiment - up to its maximum sizes in all the directions orthogonal ( $a_{1}$ and $b_{1}=1 / 3 a_{1}$ ) and parallel $\left(c_{1}\right)$ to the stretching direction. The free energy of formation of a crystallite within a parallelepiped shape (the general scheme is presented in a previous paper [18] is classically written:

$$
\begin{aligned}
\Delta \varphi\left(a_{1}, b_{1}, c_{1}, T, \lambda\right)= & 2 c_{1}\left(a_{1}+b_{1}\right) \sigma_{l}+2 a_{1} b_{1} \sigma_{e} \\
& -a_{1} b_{1} c_{1} \Delta G_{m}(T, \lambda)
\end{aligned}
$$

where $c_{1}, a_{1}$ and $b_{1}$ are the crystallite sizes in the directions (002), (200) and $(020)$ respectively. $\sigma_{\mathrm{l}}\left(0.0033 \mathrm{~J} \mathrm{~m}^{-2}\right)$ and $\sigma_{\mathrm{e}}\left(0.0066 \mathrm{~J} \mathrm{~m}^{-2}\right)$ are the lateral and chain end surface energies. Their values were deduced from the Thomas-Stavley relationship [20] and from the assumption that $\sigma_{\mathrm{e}}$ is equal to twice $\sigma_{l}$ as proposed in a previous paper [18]. Indeed, if the formation of a nucleus needs chain extension, the stretching ratio at which it is formed is far from the chains limit extensibility. Therefore, it seems reasonable to consider that the crystallite nucleus is made of both extended and folded chains. As the precise characterization of this nucleus is technically impossible, and from the different calculations we have tried to predict the experimental data, we have chosen to take a $\sigma_{\mathrm{e}}$ over $\sigma_{l}$ ratio corresponding to that of a nucleus made of folded chains, like in Thermal Induced Crystallization (TIC). 
The melting Gibbs free energy of a stretched polymer at a temperature $T$ is:

$\Delta G_{m}(T, \lambda)=\Delta H_{m}\left(\frac{T_{m, \infty}-T}{T_{m, \infty}}\right)+\frac{\nu R T}{2}\left(\lambda^{2}+\frac{2}{\lambda}-3\right)$

In the following, the term on the left is classically named enthalpic energy and the term on the right the strain (or entropic) energy. The expression of the strain energy assumes Gaussian and affine deformation of the chains [21]. The melting enthalpy $\Delta H_{m}$ is equal to $6.1 \times 10^{7} \mathrm{~J} \mathrm{~m}^{-3}$ [22], the melting temperature of the infinite crystal in the undeformed state $T_{m, \infty}$ is equal to $35.5^{\circ} \mathrm{C}$ [23], $\nu$ is the average network chain density of the material deduced from swelling measurements (in mol cm$\left.{ }^{-3}\right), R\left(8.314 \mathrm{~J} \mathrm{~mol}^{-1} \mathrm{~K}^{-1}\right.$ ) the constant of the perfect gazes. Equation (4) corresponds to the simplified expression of the Gibbs free energy developed by Flory [24]. According to his theory, the growth of crystallites should decrease the strain energy of the remaining amorphous part of the chain and therefore $\nu$ and $\lambda$ should be affected by the increase of crystallinity. This aspect is not taken into account in our simplified equation.

\subsection{Conditions for nucleation and melting}

In quasi-static conditions, crystallization occurs during stretching (or cooling in the deformed state) within a time equal or lower than the experimental time, i.e. when the nucleation barrier is low enough to be crossed. This nucleation barrier is given by:

$\left.\frac{\partial \Delta \varphi}{\partial a_{1}}\right)_{a_{1}=a^{*}, \lambda=\lambda_{c}, T=T_{c}}=0$

$a^{*}=\frac{4 \sigma_{l}}{\Delta G_{m}\left(T_{c}, \lambda_{c}\right)}$

were $a^{*}$ is the critical radius of nucleation. Applying the same equations to $c_{1}$, and assuming that $a^{*}=b^{*}, c^{*}$ is found equal to $a^{*} \sigma_{e} /$ $\sigma_{l}$.

The boundary conditions for the melting of the crystallites are reached when the amorphous and crystalline phases are in an equilibrium state, i.e. when the free energy of crystallization is equal to zero:

$\Delta \varphi)_{a_{s a t}, \lambda_{m}, T_{m}}=0$

As justified in a previous paper [18], the crystallites are assumed to reach their saturation size instantaneously once they have nucleated. $a_{\text {sat }}$ is deduced from the following equation:

$a_{s a t}=\frac{8 \sigma_{l}}{\Delta G_{m}\left(T_{m}, \lambda_{m}\right)-\frac{2 \sigma_{e}}{c_{s a t}}}$

where $\Delta G_{m}$ is the Gibbs free energy reached at the melting equilibrium, i.e. at $\lambda=\lambda_{m}$ and $T=T_{m}$

\subsection{Distribution of crystallite populations}

On the basis of experimental observations [18], we suggested that strain induced crystallization of NR during stretching performed at low strain rate (close to "quasi-static" condition) and room temperature is the result of the successive nucleation of crystallite populations. Their melting during unloading at same temperature is due to their progressive disappearance in the reverse order. The different parameters associated with each crystallite population were deduced by combining in situ WAXS analysis and the thermodynamic approach, the basis of which was presented above. They were obtained from the discretization of the curves of the crystallinity index and crystallite size versus the stretching ratio (Fig. 12 in the Appendix). Each crystallite population is made of chains with a specific network density $\nu_{i}$, reach a critical radius $a_{i}^{*}$ at the stretching ratio at SIC onset $\lambda_{c, i}$ and instantaneously grows up to the saturated radius $a_{\text {sat }, i}$. These crystallite populations then disappear as soon as the melting stretching ratio $\lambda_{m, i}$ is reached. For sake of simplicity, the present model assumes that the local stretching ratios $\lambda_{c, i}$ and $\lambda_{m, i}$ are equal to the macroscopic stretching ratio, whatever the considered crystallite populations [18].

The crystalline fraction associated with each population $\mathrm{Cl}_{i}$ was also deduced. All the parameters values are listed in Table 1 in the Appendix. For more details on the procedure used for this estimation, the reader can refer to the previous paper [18]. With this approach, the first populations that appear during stretching, also the most thermally stables (the last which disappear during unloading), are the smallest crystallites made of chains with the highest network density.

With the knowledge of these parameters, the model is now applied to more complex thermo-mechanical paths. In particular, the effect of the temperature is studied. The crystallization and melting temperatures are calculated for each crystallite population for a large range of stretching ratio (from $\lambda=1$ to $\lambda=6.5$ ). The model is then compared to results obtained by in situ WAXS experiments performed during a cycle above room temperature, and also during cooling and heating in the deformed state.

\section{Results and discussion}

\subsection{Crystallization}

From equation (5) the energy barrier needed to crystallize a given population i can be estimated by:

$\Delta \varphi_{i}^{*}=\frac{32 \sigma_{l}^{2} \sigma_{e}}{\Delta G_{m, i}\left(T_{c, i}, \lambda_{c, i}, \nu_{i}\right)^{2}}$

We have considered that, corrected from the relative temperature variation $\left(\Delta \varphi_{i} / k_{b} T\right)$, the same energy barrier needs to be crossed so that the same population crystallizes under stretching at another temperature within the same experimental time. In other words the nucleation probability related to $\Delta \varphi_{i} / k_{b} T$ is assumed constant. Given equations (4) and (9), this means that a temperature increase, which would lead to a decrease of the enthalpic energy and thus an increase of the energy barrier, must be compensated by an increase of the entropic energy. Thus, for a given stretching ratio $\lambda_{c, i}$, one can calculate from the experimental value of $\Delta G_{i}$ (or equivalently of $a_{i}^{*}$ ), the critical temperature $T_{c, i}$ needed to crystallize the population $\mathrm{i}$, within the same experimental time. Given the temperature range explored in the study, $T_{c}$ $T$ can be approximated by one. From equation (6), the temperature of crystallization $T_{c, i}$ of a given population i is then given by:

$$
T_{c, i}=\frac{\left.T_{m, \infty} 1-\frac{4 \sigma_{l}}{\Delta H_{m} a_{i}^{*}}\right)}{\left.1-\frac{\nu_{i} R T_{m, \infty}}{2 \Delta H_{m}} \lambda_{c, i}^{2}+\frac{2}{\lambda_{c, i}}-3\right)}
$$

Room temperature $(294 \mathrm{~K})$ is the temperature at which the parameters are estimated. The calculated $\nu_{i}$ values are assumed fixed because the temperatures tested are low enough (i.e. much lower than the vulcanization temperature) to avoid any degradation of the polymer chains. 
In order to illustrate the effect of the temperature on the $\lambda_{c, i}$ values of the different populations, four $T_{c, i}\left(\lambda_{c, i}\right)$ curves are plotted in Fig. 1, including the first and the last populations which appear during stretching at room temperature (parameters for the different populations are given in Appendix). These curves represent the energetic conditions in terms of temperature and stretching ratio below which the nucleation of the concerned populations should occur within a time lower or in the range of the experimental time (stretching time at a fixed temperature or cooling time in the deformed state). It is recalled that in such description, the growth process of the crystallites is assumed to be instantaneous. The temperature of crystallization of the first population $\left(T_{c, 1}\right)$ increases the most rapidly with the stretching ratio because these crystallites nucleates in polymer zones which have the highest elastically effective crosslink density $v_{1}$, i.e. associated with the highest strain energy. As a contrary, the population associated with the lowest crosslink density (population 9) poorly depends on stretching ratio, and crystallizes at a temperature around $15{ }^{\circ} \mathrm{C}$ in the unstretched state. It is known that the time needed to allow thermal crystallization in NR sample at this temperature is much longer that the range of the experimental time of the present study. A study from Bekkehdal reported that one year is necessary to significantly crystallize a sample kept at $13{ }^{\circ} \mathrm{C}$ [25]. This contradiction is likely due to the very strong assumption used for the calculation of the couple $\left(T_{c}, \lambda_{c}\right)$ : indeed it is assumed that the energy barrier (corrected from the temperature variation) does not change with temperature as far as the enthalpic term (temperature dependent) can be compensated by the entropic term (mainly stretching dependent). This implicitly assumes a questionable identical nucleation mechanism in thermal induced crystallization and strain induced crystallization.

Whatever the questions risen by the model, it is still interesting to discuss the influence of the thermo-mechanical path on the crystallization process predicted by this approach. Path A, shown on Fig. 1, illustrates Thermal Induced Crystallization (TIC): $\lambda$ is kept equal to 1 , and the sample is cooled down from high temperature at which the material is $100 \%$ amorphous. The figure suggests that during such experiment, the first population to crystallize is made of long active chains. This is consistent with experimental results showing that thermal crystallization is hampered by the presence of crosslinks $[2,3,5]$.

A classical way to crystallize a sample at room temperature consists in stretching it (cf. path B in Fig. 1). Another way is to

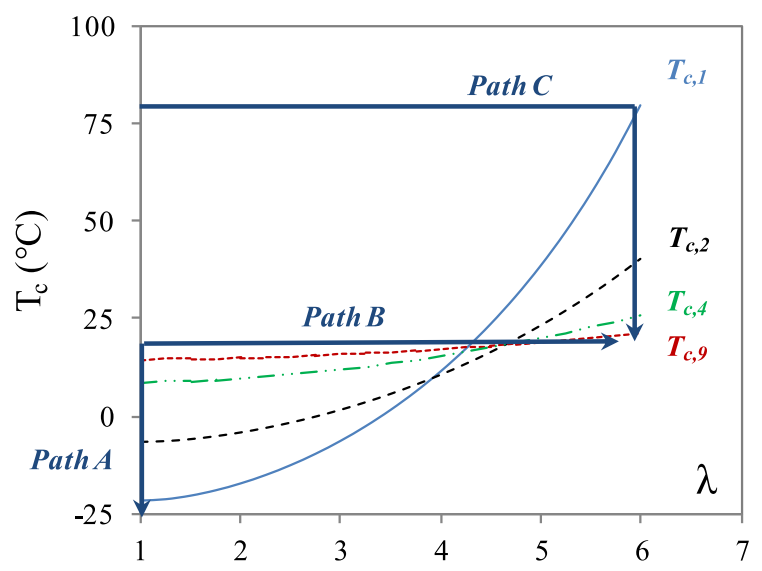

Fig. 1. Crystallization temperatures $T_{c, i}$ as a function of the stretching ratio for several crystallite size populations: $T_{c, 1}$ (solid line), $T_{c, 2}$ (dotted line), $T_{c, 4}$ (dash dotted line) and $T_{c, 9}$ (points). The arrows represent the thermo-mechanical paths illustrating the different crystallization processes: TIC (path A), SIC during stretching at room temperature (path B) and SIC during cooling at a fixed stretching ratio $\lambda=6$ (path C). stretch the sample at high temperature (so that it stays amorphous) and cool it down at a fixed $\lambda$ (path $\mathrm{C}$ ). Within these conditions, successive nucleation of the crystallite populations is promoted by increasing the stretching ratio (from left to right) or by decreasing the temperature (from top to bottom). The model suggests that for both paths, the crystallization process as well as the final crystalline state will be the same. Note that this is likely true when the sample is crystallized from a totally amorphous state. In the inverse case, e.g. when a cycle is performed above the melting stretching ratio, nucleation is enhanced by the memory of the chains alignment [18]. Obviously, such observation cannot be predicted by the present thermodynamic approach.

In Fig. 1, the curves $T_{c, i}(\lambda)$ all cross close to a singular point of coordinates $\lambda_{c}$ around 4 and $T_{c}$ around room temperature. Below this point, crystallization is eased in the domains made of long chains leading to largest crystallites, as already observed in TIC process (path A), and therefore the crystallization should proceed from successive nucleation of populations associated with active chains of decreasing size. The inverse should occur for experimental conditions in the domain of $\lambda$ and $T$ higher than 4 and room temperature respectively.

In situ WAXS experiments are now presented and compared with the present model, in particular with the thermo-mechanical paths B and C (Fig. 1).

\subsubsection{Type B path}

Two cyclic tests are carried out at relatively slow strain rate $\left(4.2 \times 10^{-3} \mathrm{~s}^{-1}\right)$ from the unstretched state up to $\lambda=6$ and two temperatures $\left(21^{\circ} \mathrm{C}\right.$ and $\left.50^{\circ} \mathrm{C}\right)$. Such conditions correspond to path B type experiments (horizontal arrow on Fig. 1). The test carried out at room temperature is the one used in the previous paper to provide the reference $\mathrm{CI}$ curve, from which the parameters values associated with each population were deduced. In Fig. 2a, CI is plotted as a function of the stretching ratio for both tests (only the stretching phase is discussed at this stage). SIC appears at $\lambda_{c}=4.3$ and 5.4 for $21^{\circ} \mathrm{C}$ and $50{ }^{\circ} \mathrm{C}$ respectively. As shown in Fig. $2 \mathrm{~b}$, the crystallite sizes $L_{200}, L_{120}$ progressively increase with CI during the loading phase and the $L-\mathrm{CI}$ curves of both tests are superimposed. Such a very interesting result reinforces our theoretical scheme: an increase of the temperature only delays the crystallization of the populations without changing their order of appearance. They still probably appear from the smallest to the largest independently of the temperature tested.

To confirm our interpretation, the experimental values of $\lambda_{c}$ extracted from Fig. 2a are compared to the crystallization curve of the first population (Fig. 3). Values of $\lambda_{c}$ from Rault's experiments performed on vulcanized NR in similar experimental conditions are also added [9]. Experimental data and $T_{c, 1}$ curve are in good agreement. This comforts the assumption that the nucleation barrier that needs to be crossed to crystallize the first population is constant (in the temperature range tested, and when corrected from the relative temperature variation). Within the frame of the model, this confirms that a decrease of the entropic energy must be compensated by an increase of the strain energy to initiate crystallization within the same experimental conditions.

\subsubsection{Type C path}

An NR sample is now stretched at $80{ }^{\circ} \mathrm{C}$ and at low strain rate $\left(4.2 \times 10^{-3} \mathrm{~s}^{-1}\right)$ up to $\lambda=6$. This temperature is chosen high enough to avoid crystallization during stretching. The sample is then cooled down to room temperature (following the type $C$ path, as illustrated in Fig. 1). The experimental device does not allow controlling the cooling rate of the sample. Cooling is obtained by opening the furnace and letting the sample cooling down to room temperature. Fortunately, the time needed to naturally reach room temperature 

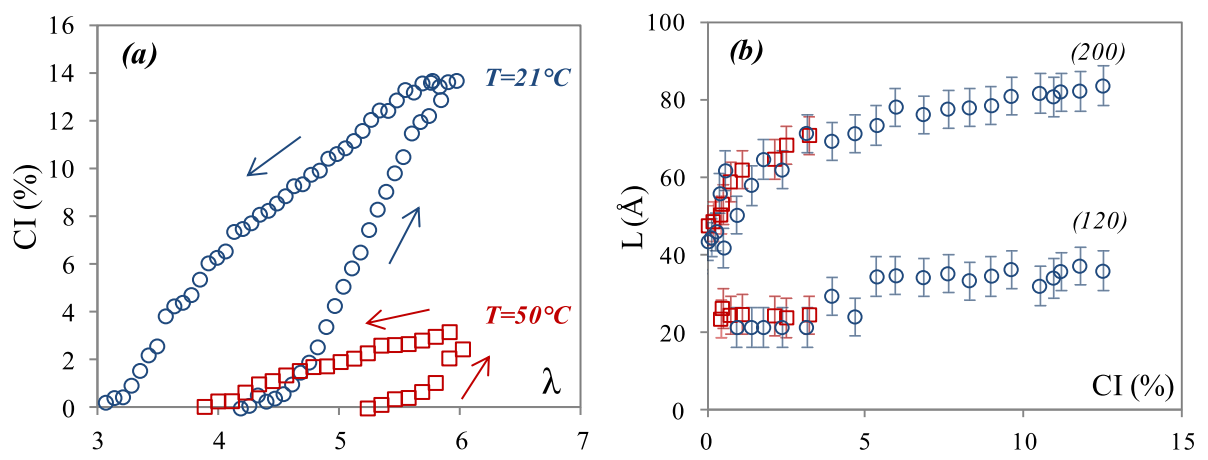

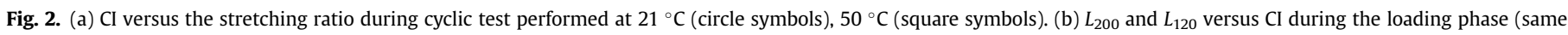
legend than in Fig. 2a).

$(\sim 2000 \mathrm{~s})$ is in the same range as the time given to the crystallization to occur during stretching ( 1000 s). The evolution of the average size of the crystallites $L_{120}$ and $L_{200}$ are plotted as a function of CI (Fig. 4). In order to provide a comparison, the $L-\mathrm{CI}$ curve corresponding to the stretching up to $\lambda=6$ at room temperature is added on the same graph (type B path). The thermo-mechanical histories between both tests are different but the final experimental conditions are chosen identical $\left(\lambda=6\right.$ and $\left.T=21^{\circ} \mathrm{C}\right)$. Fig. 4 shows that SIC during cooling (type $C$ path) leads to an increase of the average crystallite size in the same way as for the classical SIC process (type B path). Moreover, the final values of $\mathrm{CI}$ and of the average crystallite size are similar.

The superimposition of the $L-\mathrm{CI}$ curves presented in Fig. 4 also suggests that crystallite populations involved in SIC are successively activated in a similar way if the temperature decreases or if the stretching ratio increases. Within the frame of the present model, this suggests an equivalent effect of an increase of the strain energy and decrease of the enthalpic energy on SIC. Fig. 5 shows the evolution of the experimental values of $\mathrm{CI}, L_{200}$ and $L_{120}$ as a function of the crystallization temperature for the path $C$ type experiment (cooling from $80^{\circ} \mathrm{C}$ to $21^{\circ} \mathrm{C}$ at $\lambda$ equal to 6 ). $\mathrm{Cl}$ increases when temperature decreases with an initial slope of $-0.5 \%{ }^{\circ} \mathrm{C}^{-1}$. This is consistent with experiments carried out on natural rubber [1,26] and synthetic rubber [10] cooled down within a similar procedure. To go a step further in the analysis, the data are compared to the model predictions. $\mathrm{CI}$ at a given temperature is simply deduced following the path $\mathrm{C}$ in Fig. 1. This one is calculated by summation of the $\mathrm{Cl}_{\mathrm{i}}$ contribution of each population that nucleates when its $T_{c, i}$ is reached $\left(\mathrm{CI}_{\mathrm{i}}\right.$ values are given in Appendix).

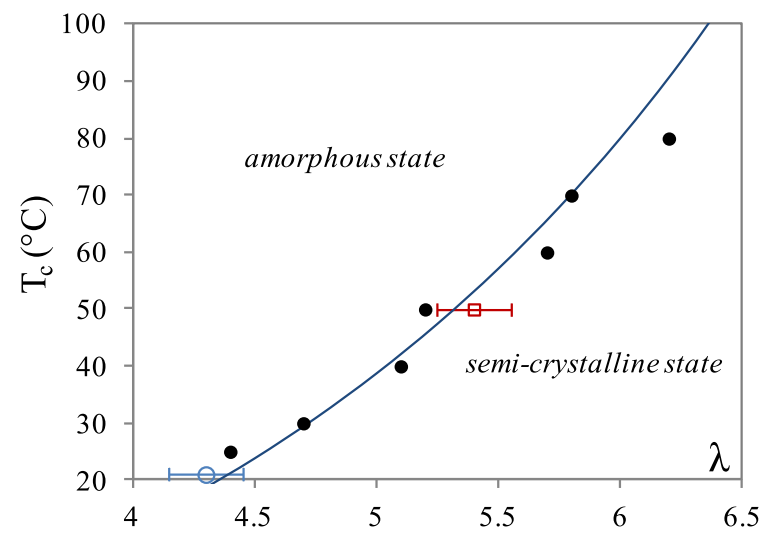

Fig. 3. Temperature of crystallization versus $\lambda$ deduced from cyclic tests at $21{ }^{\circ} \mathrm{C}$ (unfilled circle symbol) and $50{ }^{\circ} \mathrm{C}$ (unfilled square symbol). Rault's data [9] are added (filled circle symbols). $T_{c, 1}$ curve extracted from the model is given in solid line.
Within the frame of the model, the average crystallite size corresponds to a given $\mathrm{CI}$ (refer to the values in Appendix). For a given temperature, it is thus directly deduced from the knowledge of the cumulative $\mathrm{Cl}_{\mathrm{i}}$ values. Model prediction and experimental data are compared in Fig. 5 and are in reasonable agreement. The consideration of several crystallite populations finally allows us quantifying the similar effects of temperature and stretching on SIC.

\subsection{Melting}

From equation (3), one can define the thermodynamic conditions for the melting of each crystallite population:

$$
\begin{aligned}
\Delta G_{m, i} & \left.=\frac{\nu_{i} R T_{m, i}}{2} \lambda_{m, i}^{2}+\frac{2}{\lambda_{m, i}}-3\right)+\frac{\Delta H_{m}\left(T_{m, \infty}-T_{m, i}\right)}{T_{m, \infty}} \\
& =\frac{8 \sigma_{l}}{a_{s a t, i}}+\frac{2 \sigma_{e}}{c_{s a t, i}}
\end{aligned}
$$

This equation assumes that the melting occurs only when the crystallite population has nucleated and reached its saturation size (the growth is still considered as instantaneous). This melting occurs instantaneously and completely as soon as both temperature $T_{m, i}$ and stretching ratio $\lambda_{m, i}$ are reached. Different couples of values $\left(\lambda_{m, i}, T_{m, i}\right)$ correspond to the melting conditions. The melting temperature of each population can be expressed as a function of the melting stretching ratio $\lambda_{m, i}$ :

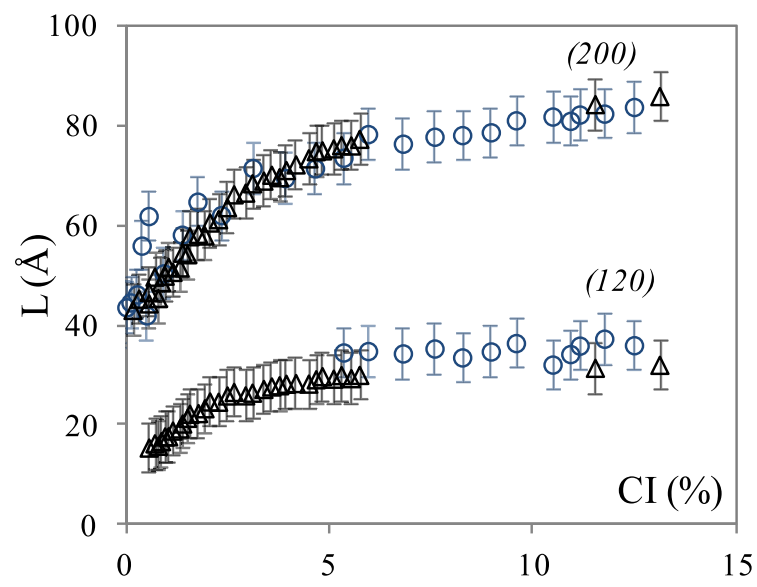

Fig. 4. $L_{200}$ and $L_{120}$ versus $\mathrm{Cl}$ for crystallization at room temperature during loading (Path B, circle symbols) and crystallization during cooling from $80^{\circ} \mathrm{C}$ to $21{ }^{\circ} \mathrm{C}$ at $\lambda=6$ (Path $\mathrm{C}$, triangle symbols). 

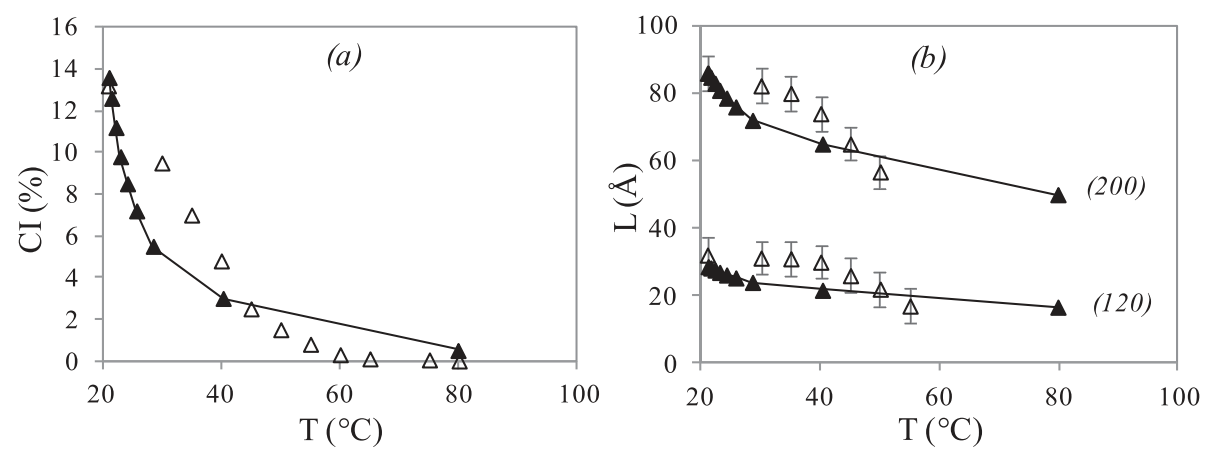

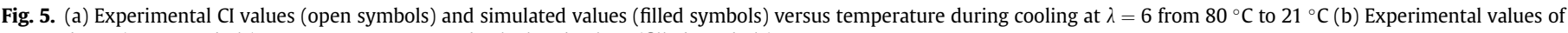
$L_{200}$ and $L_{120}$ (open symbols) versus temperature and calculated values (filled symbols).

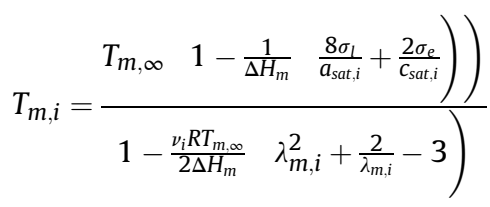

Note that in the case of an infinite crystal, equation (12) can be rewritten as:

$$
T_{m, i}=\frac{T_{m, \infty}}{1-\frac{\nu_{i} R T_{m, \infty}}{2 \Delta H_{m}}\left(\lambda_{m, i}^{2}+\frac{2}{\lambda_{m, i}}-3\right)}
$$

This equation, which assumes affine deformation, is similar to the one proposed by Krigbaum et al. [27] $T_{m, i}\left(\lambda_{m, i}\right)$ curves are calculated for each crystallite population and some of them plotted in Fig. 6 (for $\mathrm{i}=1,2,3,4$ and 9). The curves show the same trend as the $T_{c, i}$ curves. In particular, a singular point $\left(\lambda_{\mathrm{m}} \sim 3, T_{\mathrm{m}} \sim 21{ }^{\circ} \mathrm{C}\right)$ separates two melting processes.

Let us now consider the case of a NR sample crystallized at $\lambda_{c}$ and $T_{C}$ below the singular point (e.g. $\lambda_{c}=1, T_{C}=-25{ }^{\circ} \mathrm{C}$ ). In these conditions, all populations have crystallized (cf. Fig. 1). The sample is then heated (path A, Fig. 6), and all the $T_{m, i}$ curves are successively crossed from $i=1$ to $i=9$. Thus, the most thermally stable populations in the unstretched state are the largest one, and correspond to the zones of the sample with the lowest elastically effective network chains. This is in agreement with experimental results from literature [6]. The melting temperature deduced from

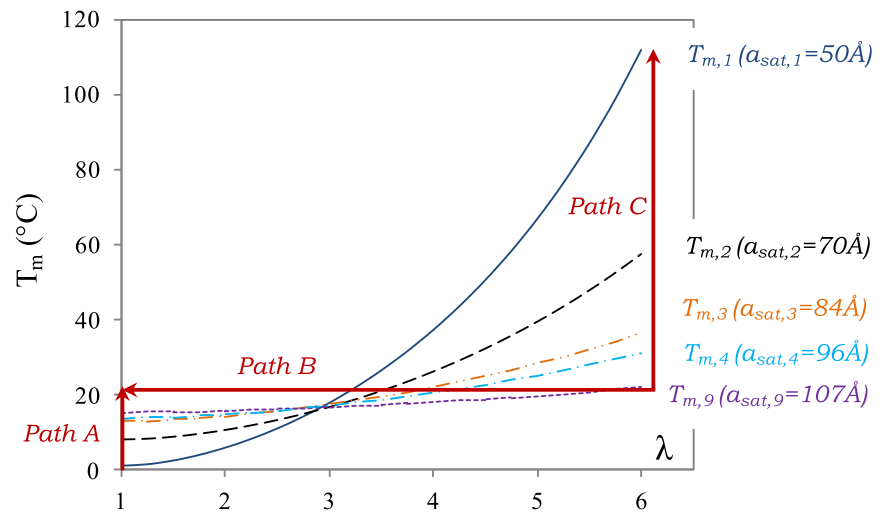

Fig. 6. Melting temperature $T_{m, i}$ as a function of the stretching ratio for several crystallite populations: $T_{m, 1}$ (solid line), $T_{m, 2}$ (dotted line), $T_{m, 3}$ (dash double dotted line), $T_{m, 4}$ (dash dotted line) and $T_{m, 9}$ (points). The arrows illustrate the paths followed by the different melting processes: melting in the unstretched state (path A), melting during unloading at room temperature (path B) and melting during heating at a fixed stretching ratio $\lambda=6$ (path C).
DSC (Differential Scanning Calorimetry) measurements after cold crystallization in the unstretched state extends from $-15^{\circ} \mathrm{C}$ to $5^{\circ} \mathrm{C}$ for samples of NR crystallized between $-25{ }^{\circ} \mathrm{C}$ and $-15{ }^{\circ} \mathrm{C}$ [5,25,28]. Our predicted $T_{m, i}$ values are also distributed (over $15^{\circ} \mathrm{C}$ ), up to a maximum value which is $8^{\circ} \mathrm{C}$ higher than the experimental one, likely because of the strong assumptions of the model.

Above the singular point (in terms of stretching ratio and temperature), melting of the populations during unloading at a fixed temperature (path B) or during heating at a constant stretching ratio (path C) occurs in the same order (refer to arrows in Fig. 6). The first crystallite population to melt is the largest one, which are made of long chains. The last population to melt is the smallest one, and its high thermal stability is due to the large crosslink density in the polymer chains involved (as it leads to a high strain energy).

In situ WAXS experiments are now performed following the melting paths $B$ and $C$ in order to be compared with the theoretical frame presented above. Three NR samples are first stretched at $\lambda=4.3, \lambda=5.3$ and $\lambda=6$ at relatively slow strain rate $\left(4.2 \times 10^{-3} \mathrm{~s}^{-1}\right)$ and at room temperature. They are then relaxed during $5 \mathrm{~min}$ in the deformed state. The samples are finally heated in the deformed state (Path $\mathrm{C}$ type) from room temperature to the temperature of the total melting of crystallites (by $10^{\circ} \mathrm{C}$ steps, with a heating rate of $10^{\circ} \mathrm{C} \mathrm{min}^{-1}$ ). At each temperature step, the sample is maintained during $5 \mathrm{~min}$. CI and crystallite size $L_{200}$ are plotted in Fig. 7 and Fig. 8 respectively. In spite of the low strain rate during stretching, CI slightly increases at the beginning of the relaxation step at room temperature, and tends to saturate after several minutes. Such an evolution is more pronounced for the lowest stretching ratio $(\lambda=4.3)$, whereas it is negligible for the highest one. $L_{200}$ follows the same trend. Within the frame of a scenario of crystallization by successive nucleation of crystallite populations, this $\mathrm{CI}$ increase can be explained by the nucleation of populations for which critical stretching ratio $\lambda_{c, i}$ is just above the stretching ratio at which stretching is stopped. Their nucleation needs longer time as their nucleation barrier is higher. The "stabilization" is due to the fact that the energy barrier is very dependent on the stretching ratio and on the elastically effective crosslink density of the considered population. Therefore, at a fixed stretching ratio, the nucleation rate associated with crystallite population $\mathrm{i}$ tends rapidly towards zero when i increases. Moreover, it was found in the previous paper [18] that the distribution of the effective network chain density $\nu_{\mathrm{i}}$ of the crystallizing zones spreads towards high values of $\lambda_{i}$. Therefore, the nucleation energy barriers associated with each crystallite population should be distributed in the same way, which is consistent with the more important $\mathrm{Cl}$ increase during the relaxation step at the lowest stretching ratio.

Considering now the melting during heating in the deformed state, $\mathrm{CI}$ decreases and immediately reaches a plateau when the temperature stabilizes. The time of the different temperature steps is thus long enough to consider that the equilibrium state is 

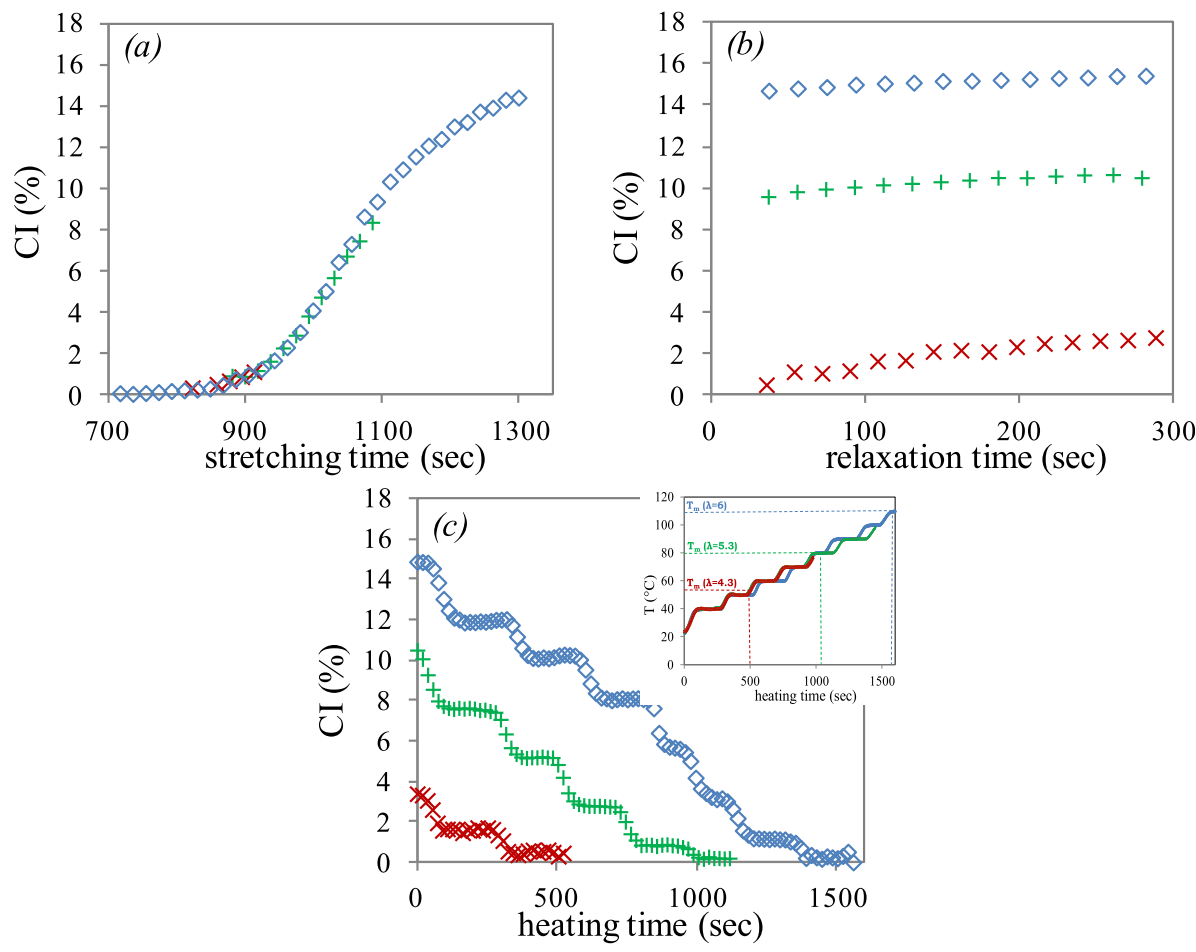

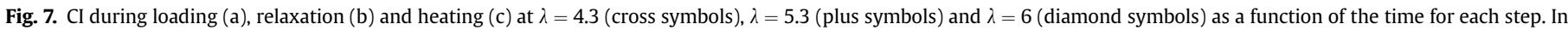
insert of Fig. $7 \mathrm{c}$ is given the evolution of the temperature during the heating step and dotted lines give the values of the melting temperature for each test.

reached. This is consistent with a recent study of Brüning [29] that showed that the rate of melting is extremely fast (around $10 \mathrm{msec}$ ), at least much higher than the heating rate of the present study. The same trend is observed for $L_{200}$, as shown on Fig. 8c. Given that the size $L_{002}$ is found constant and recalling the relation $L_{120}=1 / 3 L_{200}$, it can be easily shown that the average number of crystallites $(N=\mathrm{Cl} / V)$ decreases during the melting in the deformed state. Within the frame of the model, this is in agreement with the
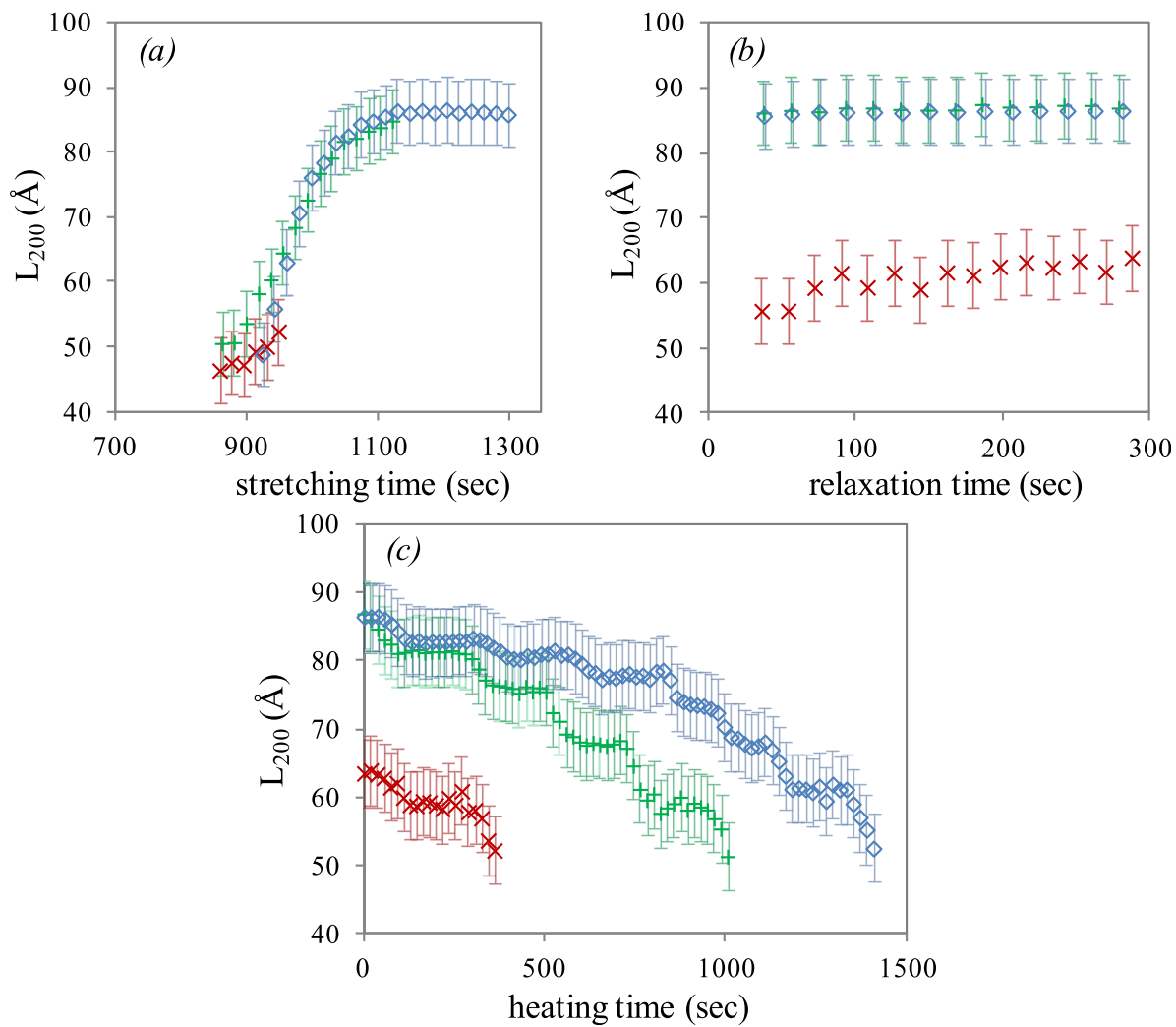

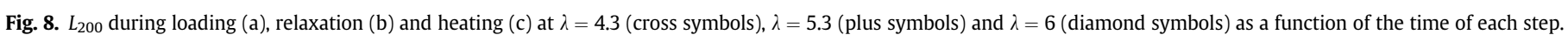




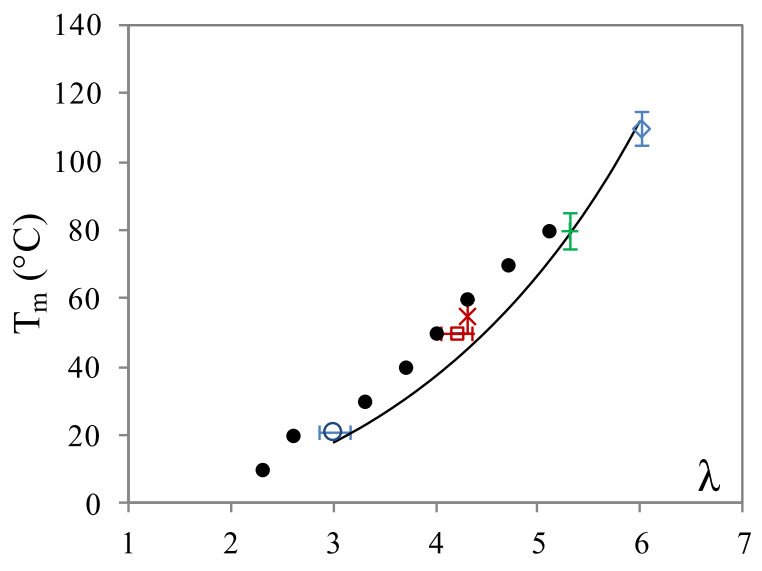

Fig. 9. Melting temperature deduced from cyclic tests at $21^{\circ} \mathrm{C}$ (circle symbol), $50{ }^{\circ} \mathrm{C}$ (square symbol) and from heating at $\lambda=4.3$ (cross symbol), $\lambda=5.3$ (plus symbol) and $\lambda=6$ (diamond symbols). $T_{m, 1}$ curve extracted from the model is added in continuous line. Experimental $T_{m}$ values from Rault's study [9] are added (filled circle symbols).

assumption that the melting, which occurs instantaneously at each heating step, involves the complete melting of several crystallite populations of decreasing size.

The temperatures corresponding to the complete melting of the crystallites (see $T_{m}$ values in the insert of Fig. 7) as a function of the stretching ratio are plotted on Fig. 9. The melting stretching ratio measured during type $\mathrm{C}$ experiments performed at $21^{\circ} \mathrm{C}$ and $50{ }^{\circ} \mathrm{C}$ (Fig. 2a) are recalled. The data from reference 9 are also added. The calculated curve of the melting temperature of the first population $\left(T_{m, 1}\right)$ is reported on the same graph, and is found in good agreement with the experimental data. This strongly supports the modeling approach used in this study.

$\mathrm{CI}$ and the average crystallite sizes $L_{200}$ deduced from the type C experiment are plotted as a function of the heating temperature in Fig. 10 and Fig. 11. Only the experimental values reached at the "plateau" after each heating step are reported. They are compared to the cumulative $\mathrm{CI}$ and the average crystallite sizes deduced from the model. $\mathrm{CI}$ is calculated as follows: when temperature reaches $T_{m, i}$, the crystallite populations i melt, $\mathrm{CI}$ is thus decreased of the corresponding $\mathrm{Cl}_{\mathrm{i}}$. As previously, the average crystallite size estimated from the model is directly deduced from the knowledge of the global $\mathrm{CI}$, and from the relationship between the average size and $\mathrm{CI}$. Given the experimental uncertainties and the different approximations and assumptions made in the model, comparison between experimental data and calculated ones are in reasonable agreement. The discrepancy mainly observed at the beginning of the temperature ramp is likely due to a slight increase of the saturated size of the crystallites by the high stretching ratio maintained during the experiment.

\section{Conclusion}

In a previous work, a scenario was proposed to describe strain-induced crystallization (SIC) of Natural Rubber (NR) during cyclic test at $21{ }^{\circ} \mathrm{C}$ and slow strain rate $\left(4.2 \times 10^{-3} \mathrm{~s}^{-1}\right)$. It was assumed that SIC is governed by successive nucleation of crystallite populations of increasing size, which occurs at a stretching ratio depending on the effective crosslink density of the polymer zones involved. The growth was assumed instantaneous within the experimental time scale explored. During unloading, the melting would be due to the progressive disappearance of the different crystallite populations in the reverse order. In the present study we proposed to explore the consequences of this scenario when the sample is submitted to more complex thermomechanical histories. The model predicts that, at moderate stretching ratio (below $\lambda$ around 4 ) and low temperature (below room temperature), crystallization first occurs in zones with low effective crosslink densities; the size of the population nucleated first is the largest. Melting is first observed for the crystallites of the smallest size, having nucleated in zones of high effective crosslink density. Conversely, for large stretching ratios ( $\lambda$ above 4 ) and temperature above room temperature, population nucleated in zone with large effective crosslink density, i.e. the smallest one, crystallizes first; these crystallite populations are the last to melt when temperature increases, or when the stretching ratio decreases. This theoretical frame is compared with in situ WAXS experiments performed following different thermo-mechanical paths. These experiments first show that the same relationship between $\mathrm{CI}$ and the crystallite size is found, whatever the temperature of the stretching or if the sample is stretched at fixed temperature or cooled down in a stretched state. Finally, experimental crystallite sizes and crystallinity index as a function of the crystallization and melting temperature are compared to the model predictions. This comparison is encouraging and the discrepancy is easily explained by the used simplifications of the model. To conclude, our analysis evidences that the entropic and enthalpic energetic contribution must compensate each other, to allow crystallization and melting at different stretching ratios and temperatures within the same experimental time. In further works, the present description could be enriched to model and understand the effects of the temperature on SIC at strain rates much higher than the ones presented here, i.e. close to the rate of solicitation of rubber in pneumatic tire but also close to the SIC characteristic time which was recently estimated around $10 \mathrm{msec}-100 \mathrm{msec}$ [29-32].
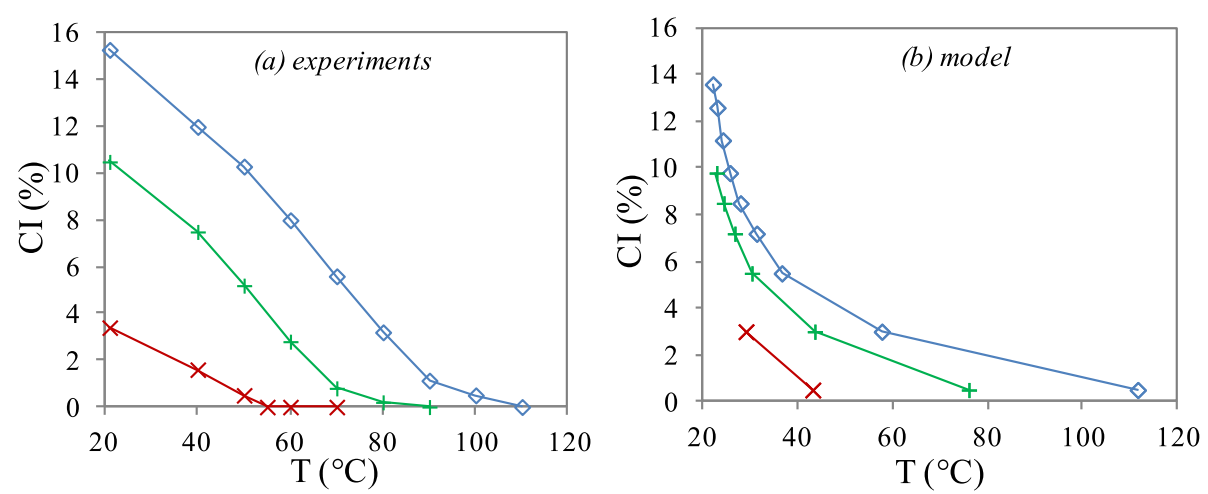

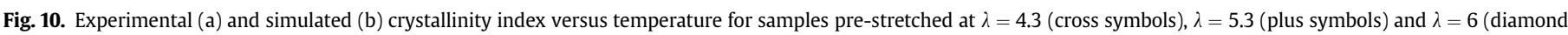
symbols) submitted to a heating ramp. 

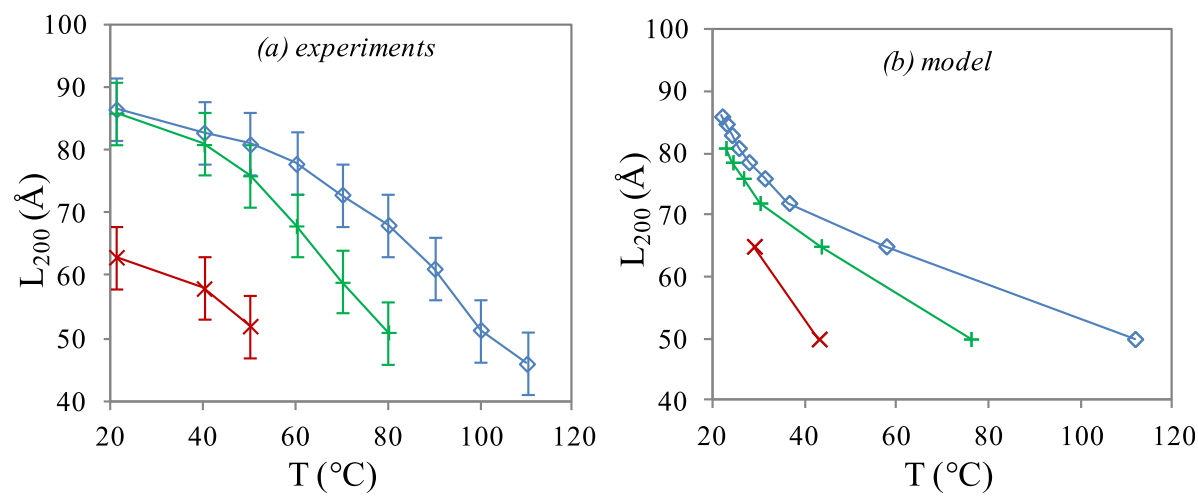

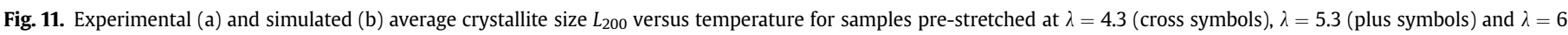
(diamond symbols) submitted to a heating ramp.

\section{Acknowledgments}

The authors are indebted to the European Synchrotron Radiation Facility (ESRF) and the local contact Dr. C. Rochas for providing the necessary beam-line time and technical assistance in the experiments on the D2AM line.

\section{Appendix}
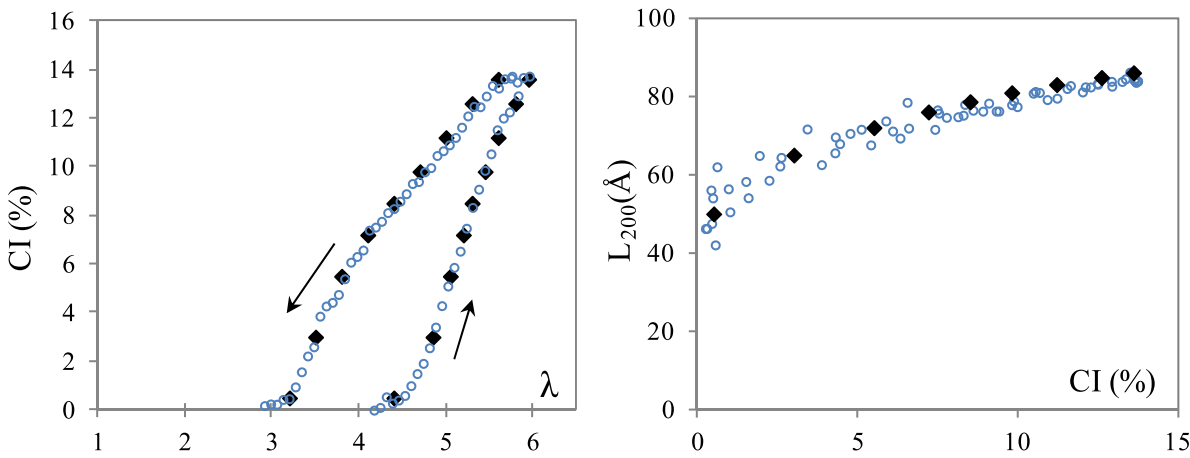

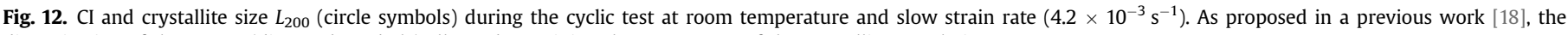
discretization of the curves (diamond symbols) allows determining the parameters of the crystallite populations.

Table 1

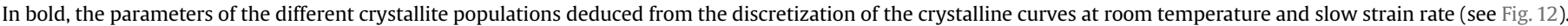

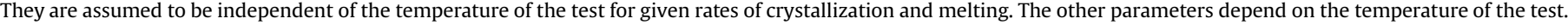

\begin{tabular}{|c|c|c|c|c|c|c|c|c|}
\hline $\mathrm{N}^{\circ}$ population & $L_{200}(\AA)$ & $a_{\text {sat }, \mathrm{i}}(\AA)$ & $\lambda_{\mathrm{m}, \mathrm{i}}$ & $\nu_{\mathrm{i}}^{\mathrm{a}}$ & $\mathrm{Cl}, \mathrm{i}$ & Cumulated CI & $\lambda_{c, \mathrm{i}}$ & $a_{\mathrm{i}}^{*}(\AA)$ \\
\hline 1 & 50 & 50 & 3.2 & 4.11 & 0.5 & 0.5 & 4.4 & 12 \\
\hline 2 & 65 & 70 & 3.5 & 2.03 & 2.5 & 3.0 & 4.75 & 17 \\
\hline 3 & 72 & 84 & 3.8 & 1.23 & 2.5 & 5.5 & 5 & 21 \\
\hline 4 & 76 & 96 & 4.1 & 0.82 & 1.7 & 7.2 & 5.2 & 25 \\
\hline 5 & 79 & 100 & 4.4 & 0.63 & 1.3 & 8.5 & 5.3 & 27 \\
\hline 6 & 81 & 103 & 4.7 & 0.51 & 1.3 & 9.8 & 5.45 & 29 \\
\hline 7 & 83 & 104 & 5 & 0.44 & 1.4 & 11.2 & 5.6 & 30 \\
\hline 8 & 85 & 105 & 5.3 & 0.38 & 1.4 & 12.6 & 5.8 & 31 \\
\hline 9 & 86 & 107 & 5.6 & 0.32 & 1 & 13.6 & 5.95 & 32 \\
\hline
\end{tabular}

${ }^{a}$ Elastically effective network chain density $\times 10^{-4} \mathrm{~mol} \mathrm{~cm}^{-3}$.

\section{References}

[1] Trabelsi S, Albouy PA, Rault J. Rubber Chem Technol 2004;77(2):303-16.

[2] Andrews EH, Owen PJ, Singh A. Proc R Soc Lond A Math Phys Sci 1971:324(1556):79-97.

[3] Gent AN. J Polym Sci 1955;18(89):321-34.

[4] Gent AN. Trans Faraday Soc 1954;50(5):521-33.
[5] Chenal JM, Chazeau L, Bomal Y, Gauthier C. J Polym Sci Pt B Polym Phys 2007:45(8):955-62.

[6] Phillips PJ, Vatansever N. Macromolecules 1987;20(9):2138-46.

[7] Edwards BC. J Polym Sci Pt B Polym Phys 1975;13(7):1387-405.

8] Andrews EH. Proc R Soc Lond A Math Phys Sci 1964;277(1370):562-70.

[9] Rault J, Marchal J, Judeinstein P, Albouy PA. Macromolecules 2006;39(24): 8356-68. 
[10] Toki S, Sics I, Hsiao BS, Tosaka M, Poompradub S, Ikeda Y, et al. Macromolecules 2005;38(16):7064-73.

[11] Albouy PA, Marchal J, Rault J. Eur Phys J E 2005;17(3):247-59.

[12] Miyamoto Y, Yamao H, Sekimoto K. Macromolecules 2003;36(17):6462-71.

[13] Rauline R. US. Patent, 5, 227, 425, Michelin, 1993.

[14] Flory PJ, Rehner J. J Chem Phys 1943;11(11):521-6.

[15] Chenal JM, Chazeau L, Guy L, Bomal Y, Gauthier C. Polymer 2007;48(4): 1042-6.

[16] Mitchell GR. Polymer 1984;25(11):1562-72.

[17] Trabelsi S. Thesis. Paris 11: Orsay; 2002.

[18] Candau N, Laghmach R, Chazeau L, Chenal JM, Gauthier C, Biben T, et al. Macromolecules 2014;47(16):5815-24.

[19] Sperling LH. Introduction to physical polymer science. 2005. Wiley. com.

[20] Hoffman JD, Davis GT, Lauritzen Jr JI. Treatise on solid state chemistry. 1976. New York.
[21] Treloar LRG. The physics of rubber elasticity. UK: Oxford University Press; 1975

[22] Kim HG, Mandelkern L. J Polym Sci Part A 2 Polym Phys 1972;10(6):1125-33.

[23] Dalal EN, Taylor KD, Phillips PJ. Polymer 1983;24(12):1623-30.

[24] Flory PJ. J Chem Phys 1947;15(6):397-408.

25] Bekkedahl N, Wood LA. Ind Eng Chem 1941;33:381-4.

[26] Trabelsi S, Albouy PA, Rault J. Macromolecules 2003;36(24):9093-9.

[27] Krigbaum WR, Roe RJ. J Polym Sci Part A General Pap 1964;2(10P):4391-414.

[28] Gent AN, Kawahara S, Zhao J. Rubber Chem Technol 1998;71(4):668-78.

[29] Bruning K, Schneider K, Roth SV, Heinrich G. Macromolecules 2012;45(19): 7914-9.

[30] Tosaka M, Senoo K, Sato K, Noda M, Ohta N. Polymer 2012;53(3):864-72.

[31] Candau N, Chazeau L, Chenal JM, Gauthier C, Ferreira J, Munch E, et al. Polymer 2012:53(13):2540-3

[32] Albouy PA, Guillier G, Petermann D, Vieyres A, Sanseau O, Sotta P. Polymer 2012;53(15):3313-24. 